\title{
Picokatal per Liter
}

National Cancer Institute

\section{Source}

National Cancer Institute. Picokatal per Liter. NCI Thesaurus. Code C122222.

A unit of catalytic activity concentration defined as activity equal to a picokatal per one liter of the system volume. 